Interactive comment on "Mobile-Platform Measurement of Air Pollutant Concentrations in California: Performance Assessment, Statistical Methods for Evaluating Spatial Variations, and Spatial Representativeness" by Paul A. Solomon et al.

Paul A. Solomon et al.

charles.Id.blanchard@gmail.com

Received and published: 24 March 2020

Interactive comment on "Mobile-Platform Measurement of Air Pollutant Concentrations in California: Performance Assessment, Statistical Methods for Evaluating Spatial Variations, and Spatial Representativeness by Paul A. Solomon et al. 
Thank-you for your thorough review. We summarize our responses to your questions and suggestions here. We will add these responses at appropriate places in the revised manuscript or in the supplement. Please note the new paragraph that we propose to add to the introduction, which has been posted as "AC1 Authors' general response." We organize our responses by the line numbers noted in the review under the two review categories

Interactive comment

Specific Comments:

Line 17: The time frame is misleading. Should indicate a few intensive (i.e. week to month long) campaigns were performed between May 2016 and September 2017.

Revise to: On-road measurements of air quality were made during a series of sampling campaigns between May 2016 and September 2017 at high. . .

Lines 20-21: Lifetime of $\mathrm{NO} 2$ is hours and $\mathrm{O} 3$ is $>20$ days in the troposphere. Observing the diurnal cycle and weekday weekend trends may be more appropriate than looking at a fortnight.

Our focus was on characterizing spatial rather than temporal variations. As noted in the proposed new paragraph, the cars drive weekdays between $\sim 9$ a.m. and 5 p.m. The sampling regime therefore does not permit weekday/weekend comparisons nor does it lend itself to fully characterizing diurnal cycles.

Line 22: In-situ instrument or research-grade instruments. I'm sure they mean their instrument package.

Revise to: ... research instruments located within stationary vehicles...

Line 31: Percentages of what? Concentration deltas?

Revise (here and at line 424) to: 1-km scale differences in NO2 and $\mathrm{O} 3$ concentrations up to $117 \%$ and $46 \%$, respectively, of mean values

Line 75: Concentration decay rate from a point source will be highly variable and based 
on several meteorological parameters.

Revise to: ...could help establish concentration decay rates of mobile emissions with AMTD distance...

Line: 109-110: What are the limitations of overnight calibration when cars are parked next to each other?

Interactive comment

We do not understand this question. Lines $109-110$ simply state that we used the QD1 data because QD1 data sets included the time periods when the cars were parked next to each other, whereas such times had been filtered out of the QD2 data set. The instruments were switched from vehicle to line power when parked.

Lines 106,109/110, 113: The first lines seem to imply the mobile platform intercomparison was made overnight, 113 implies it may have been only a short period ( $5 \mathrm{~min}, 30$ min), the SI from their Apte et al. indicates it was several hours overnight. Not sure about an intercomparison in a parking garage either, especially if it was during a time when vehicles were entering or leaving (cold starts vs operating temp emissions).

Revise to: ... with additional sampling occurring while vehicles were parked in San Francisco and Los Angeles before ( $~ 6-9$ a.m.) and after $(\sim 5-10$ p.m.) driving periods.

The vehicles are parked away from traffic in the San Francisco parking garage. They were parked overnight in a small ( $\sim 10$ car) lot in Los Angeles.

Line 119: Was the audit the same as is done with FRM/FEM monitors via the National Performance Audit Program (NPAP)?

We will provide more detail to reduce possible confusion as to how the audits were accomplished.

Line 122: In table 4, there needs to be an explanation of the scales and how EPA initially established each sitting.

Printer-friendly version

Discussion paper 
We will add citation to Appendix D to Part 58 - Network Design Criteria for Ambient Air Quality Monitoring (https://www.law.cornell.edu/cfr/text/40/appendixD_to_part_58). The EPA scales are defined in Footnote 1 of Table 4.

Line 125: Suggested to add sentence or phrase to cover why the other stations were not used.

Revise lines 119 - 125 to: During the Los Angeles sampling, the South Coast Air Quality Monitoring District (SCAQMD) conducted calibration checks when the sampling vehicles were parked adjacent to stationary air quality monitoring sites (Table 3). The SCAQMD also prepared 1-minute resolution data files for measurements made at these and other stationary air quality monitoring sites (Table 4; see also location map, Figure S1). Data from one of the dates and locations (LAXH, September 20, 2016) were suitable for collocated comparison with mobile measurements (Table 3). The stationary-monitor data from W710 consisted only of 1-hour resolution PM2.5 mass (Table 4), which was not measured by the mobile platforms, and no data were provided for the Santa Clarita site (Tables 3).

Line 137: Do the monitoring stations use the Network Time Protocol? If not, address discrepancies this may cause in measurement comparisons.

We used time series plots, such as Figure S5, to confirm the alignment of mobile and station minima and maxima. The results indicate that any discrepancies are less than the 1-minute averaging times.

Line 164: What is the impact of wind and GPS location uncertainties on data collected while stationary?

When cars were parked adjacent to each other, we do not expect GPS location uncertainties or variations in wind sped or direction to impact the side-by-side comparisons.

Sec. 2.3: Are there multiple $\mathrm{BC}$ and $\mathrm{CH} 4$ instruments or just one that was moved between cars? I'm assuming this was done because of inlet restrictions.

AMTD

Interactive comment
Printer-friendly version

Discussion paper 
One car was equipped with a BC instrument and one car was equipped with a $\mathrm{CH} 4$ instrument. There are three cars, though. The second $\mathrm{BC}$ and $\mathrm{CH} 4$ instruments were used by Apte et al. (2017) in their study. Since all vehicles parked in the same San Francisco garage, there were two $\mathrm{BC}$ and two $\mathrm{CH} 4$ instruments available for the sideby-side parked comparisons (line 180, Tables 5 and 6).

Line 173: Where are the reported variabilities of the paired differences shown?

Interactive

comment

These are reported in "Results and Discussion," rather than "Methods" (Section 3.2, Table 9).

Sec. 2.3: Was CARB contacted to ensure $\mathrm{BC}$ and $\mathrm{CH} 4$ observations were not present at sites? Some EPA sites have but don't advertise these observations.

For the field comparisons, we worked with South Coast Air Quality Management District staff, who operate the air quality monitors and are familiar with all measurements made.

Lines: 247-249: Comparing different regions during different time periods without a detailed study of the meteorology is misleading if talking about local or neighborhood scales. Here are the climatological winds near San Joaquin Valley for March and November using data between 1973-2019 at Buchanan Field Airport in Concord, CA. Figure 1 shows the month of March may be experiencing inflow from the Chevron processing plant in Richmond and dust (Coarse mode, not reported) from Dutra Materials quarry in McNears Beach, while to a much lesser extent in November (Figure 2). Since the data is presented as mean concentrations during the sampling periods, l'd bet the baseline PN concentrations are different for the two months.

Lines $247-260$ provide a summary overview of the measurements. These are useful but require caveats for various reasons such as those indicated by the referee. We stated at line 250, "Although differences in the PN distributions possibly reflect spatial variability, they more likely reflect seasonal variations in PM composition" and at lines 261 - 262, "As with PN, these average concentrations likely vary due to time of year,

Printer-friendly version

Discussion paper
AMTD 
location relative to source emissions, and chemical processing." For later analyses, we focused on time-synchronous differences between measurements made by two vehicles, not averages over short-term campaigns.

Lines 251-252: The deployed optical particle counter provided five size ranges why report only the smallest, then reference a paper regarding a measured size bin that was not reported in the paper?

Reasons for focusing on the smallest size fraction were explained in the previous paragraph, lines 240-246.

Lines 246-262: I'm not sure this section is representative and should be included here and should likely be absorbed by the following sections.

As noted previously, this paragraph provides a summary overview of the measurements but is not the basis for our analyses of spatial variability.

Line 265: Typo. ... vehicles drove in the Los Angeles

Revise by removing "the"

Line 270: Why was the mean relative difference between the two calibrations so high? An absolute difference of $5 \%$ NPAP would require corrective actions. The calibration gases and flow meters used should be traceable to NIST for re-evaluation.

We do not see large $(>5 \%)$ differences between the internal and external calibration checks in Table 8. The invalidating limits for the South Coast Air Quality Management District's weekly calibration checks are $7 \%$ for $\mathrm{O} 3$ and $10 \%$ for $\mathrm{CO}, \mathrm{SO} 2$, and NOx, warning limits are $5 \%$ for $\mathrm{O} 3$ and $7 \%$ for $\mathrm{CO}$, SO2, and NOx (Table 2.4, https://ww3.arb.ca.gov/aaqm/qa/pqao/repository/district_sops/south_coast/quality_assurance/q

Sec. 3.2: When comparing inter-vehicle observations were the vehicles traveling the same route (i.e. following each other) or just driving the same neighborhoods and passing by each other? 
The cars generally followed different routes, such as those illustrated in Figure 7 or in Figures S3, S6 - S8, and S10 - S15. When the cars traveled a route segment together (e.g., Figure S15), they traveled "caravan style", keeping each other in sight but not following immediately one behind the other.

Line 294: Were the vehicles were running during the LAXH comparison or were the instruments moved to shelter power and the vehicle engines shutoff?

The instruments were switched from vehicle to line power in the parking garage or parking lot but this option was not logistically practical for the one-day comparison at LAXH.

Sec. 3.3: Last sentence of section, $\mathrm{CH} 4$ emissions from vehicles is extremely small (something like $<0.2 \%$ of anthropogenic emissions) and the lifetime of NO very short. This statement needs a citation, or it needs to be removed.

Add citation: Nam et al., ES\&T, 2004 "We recommend the use of an average emission factor for the U.S. on-road vehicle fleet of (g of $\mathrm{CH} 4 / \mathrm{g}$ of CO2) $)=(15 \pm$ 4) $\times 10-5$ and estimate that the global vehicle fleet emits $0.45 \pm 0.12 \mathrm{Tg}$ of $\mathrm{CH} 4 \mathrm{yr}-1$ ( $0.34 \pm 0.09 \mathrm{Tg}$ of $\mathrm{C} \mathrm{yr}-1)$, which represents $<0.2 \%$ of anthropogenic $\mathrm{CH} 4$ emissions." https://pubs.acs.org/doi/10.1021/es034837g. We agree that NO has a short residence time compared to $\mathrm{CH} 4$. However, a correlation between $\mathrm{NO}$ and $\mathrm{CH} 4$ will be observed when sampling fresh automotive exhaust emissions.

Sec 3.4: This section will have a very large dependence on meteorological parameters.

We agree that meteorology impacts the concentrations measured at two distant points, as do emission sources and chemical and physical processing. The last sentence of the first paragraph in section 3.4 states "The intent of the analyses in this section is to help elucidate the spatial scales over which stationary-monitor and mobile-platform data represent ambient concentrations." The analyses utilize time-synchronous differences so that each vehicle is experiencing the same meteorological conditions. 
Sec. 3.4.1: The air masses the vehicles are sampling are potentially different. An intervehicle comparison could be made in time and latitude. As it is, the comparisons are meaningless because we know the location of any vehicle at any given time and one may be sampling south of the Santa Ana Freeway and the other sampling all three major N-S freeways in the area. The attached Figure 3 shows winds are between 9am and 5pm averaged over Aug 3-16. See comment above, section 3.4.

Because vehicles sample different road segments on different days and at different times of day, we compiled time-synchronous differences between the concentrations measured by two cars to remove the confounding effects of day-to-day and diurnal variability. Random differences between vehicles, such as short, intermittent exposures of one car or the other car to a high emitter or variations in wind directions, are averaged out in the FAMD statistic. In contrast, systematic car-to-car differences yield higher FAMD values. Systematic differences could occur if the instrumentation in one car was biased relative to the other car. After eliminating that source of systemic car-to-car difference through the side-by-side sampling comparisons, we can conclude that larger FAMD values (e.g., $>0.20$ or $20 \%$ ) represent spatial heterogeneity, e.g., due to the two cars sampling different neighborhoods (as indicated in Figure 6a or in Figures S6 and $\mathrm{S10}$ ). FAMD is a useful metric for evaluating the spatial scale of representativeness of stationary monitors, for example. The relationship between FAMD and distance does not, of course, indicate which neighborhoods experienced higher pollutant concentrations. For that purpose, we examined maps, such as shown in Figures S6 and S10, and photos such as those provided by the referee; we also developed the visualization shown in Figure 6. Please note also our interpretation at lines $352-355$.

Line 362: Driving near as in right past along Dowlen Dr or within $\mathrm{n}$ meters? Wilshire Blvd is $\sim 200 \mathrm{~m}$ as is Federal Ave.

Line 360 defines "near" as 0.5 to $5 \mathrm{~km}$. The routes include all areas shown in Figure S8. We will revise the text for clarity.

Interactive comment
Printer-friendly version

Discussion paper 
Line 392: What grid is used?

Nearest kilometer as calculated by conversion of latitude and longitude to UTM coordiAMTD nates.

Fig. 6: Needs legend, different colors for positive and negative intervehicle differences and FMD differences not red/blue, which were used to identify specific vehicles in the Interactive comment same figure.

We will revise this figure.

Line 424: Enhancements based on what? FAMD is comparing observations at the same time, is the enhancement based on location as stated in the paragraph before or between May 1-12?

Revise to: $1-\mathrm{km}$ scale differences in NO2 and $\mathrm{O} 3$ concentrations up to $117 \%$ and $46 \%$, respectively, of mean values

Line 440: Routes for November 16th, 2016 are not in SI but referenced in text.

Revise to: The initial drives occurred November 16 - 23, 2016 (Figure 7, November 16; see also example of drives on other days in Figures S11 - S15).

Include Line 457: Are traffic count data available?

We did not use traffic count data in our analyses but they are available.

Line 459: Enhancements compared to what, background?

Lines 459 - 460 state ". . .enhancements of pollutant concentrations in northern San Joaquin Valley cities over concentrations occurring in surrounding areas

Line 529: Enhancements based on what?

Printer-friendly version

Revise to: $1-\mathrm{km}$ scale differences in NO2 and $\mathrm{O} 3$ concentrations up to $117 \%$ and $46 \%$, respectively, of mean values 
General: Overall distance bins should be the same for all missions. Seems like all the analysis times were weekday (do Google Street View vehicles drive on weekends)?

The spatial scales of the sampling routes differed among the missions, so the distance bins also differ. As noted at line 112, measurements were made between $\sim 9$ a.m. and 5 p.m. on weekdays. We identify this as a limitation in the new paragraph posted as "AC1."

\section{TECHNICAL CORRECTIONS:}

Line 35: Suggested to add spatial variability context for pollutants to introduction as this has implications on reported uncertainties. Seems this is provided starting at about line 48 of the intro.

Lines $35-60$ provide this context. It isn't evident that reordering sentences would improve clarity.

Line 155: LOD is defined in Table 5 subtext, but not in text. Consider defining in main text.

Revise line 153 to: We calculate BC limit of detection (LOD) (see footnote 2, Table 5) using data reported...

Lines 172-174: Suggested to remove 'merge' detail, as it seems superfluous to the reader, and combine the two sentences into one focusing on temporally coincident pairing.

Revise to: Data files were merged by 1-s or 1-minute resolution times and were then used to determine time-matched paired differences, which were evaluated as functions of ambient concentration, intervehicle distance, and vehicle speed.

Lines 185, 190, 195, 200: 'Car B Difference' could be misleading. It is suggested to move the word 'Difference' to after the word 'Mean' (i.e., Mean Difference) or use wording such as 'Mean [Absolute] Difference between Car A and B' in the numerator.

Printer-friendly version

Discussion paper
Interactive

comment 
These changes will be made.

Line 206: $Z$ is not defined.

AMTD

$Z$ is simply an example variable, not a measurement. Lines $205-209$ will be replaced by simple citations.

Line 216: MD already defined in line 185.

Interactive comment

Not redefining MD, just restating for clarity, revise to: MD is used ...

Lines 211 and 222: Consistency in section references.

Capitalize in both locations.

Interactive comment on Atmos. Meas. Tech. Discuss., doi:10.5194/amt-2019-501, 2020. 